\title{
Lidil
}

Revue de linguistique et de didactique des langues

$50 \mid 2014$

Variation stylistique et diversité des contextes de socialisation

\section{Le traitement du passif dans l'enseignement du français langue étrangère}

The Use of Passive Voice in Class of French as a Foreign Language

\section{Christel Le Bellec et Badreddine Hamma}

\section{(2) OpenEdition}

\section{Journals}

Édition électronique

URL : http://journals.openedition.org/lidil/3642

DOI : $10.4000 /$ lidil.3642

ISSN : 1960-6052

Éditeur

UGA Éditions/Université Grenoble Alpes

Édition imprimée

Date de publication : 15 décembre 2014

Pagination : 191-211

ISBN : 978-2-84310-287-5

ISSN : 1146-6480

\section{Référence électronique}

Christel Le Bellec et Badreddine Hamma, « Le traitement du passif dans l'enseignement du français langue étrangère », Lidil [En ligne], 50 | 2014, mis en ligne le 15 juin 2016, consulté le 01 mai 2019. URL : http://journals.openedition.org/lidil/3642 ; DOI : 10.4000/lidil.3642 


\title{
Le traitement du passif dans l'enseignement du français langue étrangère
}

\author{
Christel Le Bellec* ${ }^{*}$ et Badreddine Hamma**
}

\begin{abstract}
RÉSUMÉ
Le présent travail vise à contribuer à l'articulation des recherches linguistiques portant sur le passif et de celles portant sur l'enseignement du français. Nous nous intéressons à certains aspects théoriques liés à quelques formes et valeurs du passif dont on ne peut faire l'économie dans l'acquisition de ce tour discursif. Cette étude vient ainsi combler certains manques relevés dans les manuels de FLE et tente de faire la lumière sur le fonctionnement du passif ainsi que sur les enjeux discursifs de son usage.

Nous nous attarderons, ainsi, sur trois points qui méritent, selon nous, la plus grande attention dans l'acte didactique : les particularités des valeurs sémantico-pragmatiques inhérentes aux constructions passives / actives; la question de l'aspect du procès actif/passif, ce qui nous amènera à tenir compte de certaines formes concurrentes, comme le passif en se faire; et enfin le problème posé par le choix de la préposition servant à introduire «l'agent» de la phrase passive (de versus par).
\end{abstract}

\section{ABSTRACT}

The main purpose of this paper is to establish how teaching French can take advantage of the results emerging from linguistic researches on the several uses of the passive voice in French. We are dealing, in the one hand, with some theoretical aspects related to some forms and uses of this kind of structure that are usually uncared for in the known handbooks of French as a Foreign Language (FLE); in the other hand, with the discursive functioning and stakes for such kind of uses.

Thus, we are dwelling on three aspects which deserve, in our opinion, a special attention in teaching French: first, we are explaining the nuances of semantic and pragmatic uses of passive and active constructions; then, we are dealing with the aspectual values of the verbs

* Université Paul-Valéry-Montpellier 3, Praxiling, UMR 5267 CNRS.

** Université d'Orléans, Laboratoire ligérien de linguistique, UMR 7270 CNRS. 
in each use. It leads us to take into account some concurrent uses such as those containing "se faire". At last, we are focusing on the Prepositional Phrase in the passive voice in order to study the distinction between the prepositions "de" and "par" ( $\approx$ by).

\section{Introduction}

Le présent travail vise à contribuer - à la suite de certains linguistes comme Le Goffic (1970) ou Leduc-Adine (1977) - à l'articulation des recherches linguistiques portant sur le passif et de celles portant sur l'enseignement du FLE. Plus précisément, nous nous proposons de fournir certains éléments théoriques jugés indispensables, liés à certaines formes et valeurs du passif. Nous nous attarderons sur les enjeux sémantico-pragmatiques sous-tendant les constructions passives/actives souvent délaissés dans les supports pédagogiques, ce qui recouvre à la fois la question de l'aspect et celle des distributions internes spécifiques à ce type de phrases. Nous discuterons les notions de base d' «agent», de «sujet» et de «patient» et des valeurs discursives de chaque forme de phrase, mais aussi celle du choix de la préposition pour introduire l'«agent» de la phrase passive. Le but est de remédier à certains amalgames constatés dans les pratiques de classe, ainsi qu'aux généralisations abusives qui ponctuent l'acquisition de ces faits linguistiques dans les manuels de FLE.

Nous commencerons par faire un état des lieux sur le traitement du passif dans plusieurs manuels de $\mathrm{FLE}^{1}$; nous nous focaliserons ensuite sur les différents aspects qui devraient, selon nous, faire l'objet d'une transposition didactique dans la conception des exercices et des leçons sur le passif et son rapport avec la forme active, ce qui incite à étudier les valeurs sémantiques et pragmatiques inhérentes à leur usage et les valeurs aspectuelles mises en jeu. Nous nous attarderons également sur le choix de la préposition comme tête du complément d'agent afin d'expliquer les implications de l'usage de par ou de sa concurrente $d e$. Pour finir, nous proposerons quelques pistes pour le travail de transposition.

1. Notons que l'examen des manuels a concerné une bonne vingtaine de méthodes de FLE récentes, mais pour ne pas alourdir la lecture de ce travail, nous ne retiendrons et commenterons, ici, que les huit cités suivants : $E G$, $G E F, G E F-N D, G P F N A, G P F-N I, N G F, N E d$ et $G P T$, qui nous semblent d'ailleurs assez représentatifs de ce qui se fait actuellement dans les manuels de FLE. 


\section{Le traitement du passif dans les manuels de FLE}

Avant de se livrer à la critique de certains aspects dans les manuels consultés, notons d'emblée que la grammaire (notamment pour le passif) y est appréhendée en relation avec d'autres formes du discours conformément à ce que l'on trouve dans la littérature sur le sujet. Ainsi, le $N E d$ rapproche le passif de l'usage du pronom on et des constructions impersonnelles :

(1) Une augmentation du carburant est prévue.

(2) On prévoit une augmentation du carburant.

(3) Il est prévu une augmentation du carburant. (NEd, 2010, p. 16)

On remarquera aussi que le recours aux exemples forgés semble reculer (cf. Max a lâché la main de Léa > ?La main de Léa a été lâchée par Max - qui a peu de chance d'apparaitre en discours de la part d'un francophone) au profit d'énoncés moins artificiels pris dans la presse. Néanmoins, des exemples incongrus continuent de faire surface çà et là dans certains manuels, exemples qui sont certes grammaticalement bien formés, mais qui sont difficiles à placer tels quels dans un échange naturel :

(4) La petite fille cueille des fleurs >? Des fleurs sont cueillies par la petite fille. (GEF-ND)

(5) Le Président de la République a pris la parole > ? La parole a été prise par le Président de la République. (Ibid.)

(6) Alain regarde avec intérêt toutes les jolies femmes qui passent $>$ Toutes les jolies femmes qui passent sont regardées avec intérêt par Alain. $($ GEF $)$

\subsection{Valeurs pragmatiques du passif}

En règle générale, les manuels de FLE ne font pas suffisamment ressortir les valeurs pragmatico-discursives propres au passif dans les sections qui lui sont consacrées. En effet, le passif sert essentiellement à topicaliser l'objet direct de la construction active, à maintenir le même élément discursif en position de topique, comme cela a largement été reconnu dans la littérature portant sur le sujet (cf. Gaatone, 1998; Rasmussen, 1996; Riegel, 1997). Ainsi, les formulations utilisées dans les espaces dédiés à la leçon ou à l'exposé de la règle sont très souvent floues ou ambiguës, et peuvent donner lieu à une double interprétation, comme le montrent les extraits suivants (nous mettons en gras les expressions en cause) : 
a. «La forme passive est une transformation qui permet de mettre en valeur le complément d'objet du verbe à la forme active» (Grammaire progressive du français, niveau avancé, CLE International, 1997, p. 84, désormais : GPFNA).

b. «Dans la phrase à l'actif, on focalise sur l'agent, sur celui qui fait l'action, et dans la phrase au passif, sur celui qui subit cette action (on l'appelle parfois “patient”)» (Grammaire expliquée du français, CLE International, 2002, p. $120: G E F)$.

c. «On préfère la forme passive quand on veut mettre en valeur le sujet du verbe» (Nouvelle grammaire du français. Cours de civilisation française de la Sorbonne, Hachette FLE, 2004, p. 105, désormais : NGF).

d. «On utilise le passif quand on met l'accent sur l'objet du verbe au lieu du sujet» (Grammaire progressive du français, niveau Intermédiaire, CLE International, 2002, p. 194, désormais : GPF-NI).

e. «Une même réalité peut s'exprimer à la "voix active" ou à la "voix passive" selon que l'on veut mettre en évidence : l'auteur, l'agent, la cause de l'action : actif; le patient qui subit, supporte, reçoit cette action : passif» (La grammaire des premiers temps, 2006, p. 111).

Les expressions mises en gras peuvent renvoyer aussi bien à la mise en valeur qui est représentée par la fonction topique, sa position initiale dans la phrase s'expliquant par sa faible charge informationnelle et par son haut degré de dynamisme discursif, qu'à la mise en valeur représentée par la fonction focus (ou rhème), une position marquant le caractère informationnellement chargé de l'élément en question, qui constitue l'information nouvelle (Lambrecht, 1994). La valeur de ces expressions nécessite donc des précisions, puisqu'elles peuvent renvoyer à deux notions opposées et/ou complémentaires.

Le manuel GPF-NI va encore plus loin en affirmant que : «L'on utilise surtout le passif pour les inventions, les lois et les évènements subis, par ex. : Un vaccin a été découvert. Une loi a été votée. Un homme a été agressé.» Ceci laisse entendre que le passif serait surtout utilisé pour parler d'entités inanimées (or les faits révèlent le contraire, cf. Gaatone (1998), entre autres) ou pour des animés subissant des évènements, escamotant de façon encore plus radicale la fonction pragmatique du passif. Ils ajoutent que pour les animés /+humains/, on utiliserait plutôt «on», et présentent ainsi la forme On m'a envoyé en mission comme préférable à J'ai été envoyé en mission. L'apparition de l'une ou l'autre dépend, de toute évidence, du contexte à l'œuvre. 
Concernant l'autre valeur pragmatique de la forme passive, à savoir la focalisation ou la rhématisation, rares sont les manuels qui mentionnent la possibilité de focaliser le complément d'agent exprimé par un pronom personnel tonique, dans une structure contrastive, $\mathrm{du}$ type : Ce livre n'a pas été écrit par lui mais par moi (voir la notion de «contraste» un peu plus loin).

Ainsi, l'exemple : Ce roman a été écrit par lui (GPF-NA) est présenté tout bonnement comme à éviter, sans mention d'une quelconque intention discursive. Il en va de même pour la $N G F$, puisqu'il est dit en remarque : «Il est préférable de ne pas employer la forme passive lorsque le complément d'agent est un pronom personnel. On dit : J'ai écrit cette lettre, plutôt que : Cette lettre a été écrite par moi.» (p. 104)

Il est vrai qu'hors contexte, il est difficile de trouver un tel exemple, mais aucune mention n'est faite sur la possibilité d'apparaitre en structure contrastive pour ce type de construction. La GPF-NI s'avère encore plus radicale en déclarant, sous une forme injonctive : «Par n'est jamais suivi d'un pronom. Dites : J'ai fait ce dessin. Ne dites pas : Ce dessin a été fait par moi.» (p. 194)

\subsection{Constructions concurrentes du passif}

La construction en se faire est régulièrement citée, aux côtés de la forme pronominale (Ce vin se boit chambré), comme une construction ayant le sens d'une forme passive ou comme une autre manière d'exprimer le passif. Néanmoins, la caractérisation qui est faite de cette construction en restreint beaucoup les possibilités d'emploi, en la mettant sous le coup d'une responsabilité du référent du sujet, qui fait que les spécificités de cette construction ne sont pas toujours suffisamment mises en lumière et n'incitent donc pas les apprenants à l'utiliser aussi fréquemment qu'elle l'est par les locuteurs francophones.

Ainsi, dans le cas des verbes à objet indirect, on sait que le passif canonique est impossible, à l'exception des verbes pardonner et obéir. La $G P F-N A$ indique que le passif est impossible pour ce type de verbes et que c'est la construction en on qui est utilisée en substitution, sans mentionner que la construction passive en se faire ou celle en se voir peuvent servir à pallier l'absence de cette construction. Sous certaines conditions, en effet, se faire acquiert une valeur de passif pour certains verbes à double complémentation : Marie s'est fait voler son ordinateur par un passant, en raison de l'impossibilité d'avoir: *Marie a été volée son ordinateur par un passant. 
De plus, la valeur sémantique de la construction en se faire est souvent biaisée en raison de la présence du verbe faire, qui perd son sémantisme causatif en tant qu'auxiliaire du passif associé au réflexif se. C'est ainsi que la $G E F$ indique que «lorsqu'on utilise la construction en se faire + infinitif, on sous-entend que le sujet porte une certaine responsabilité» (p. 124). Ainsi, Il a été injurié est présenté comme «neutre, on ne sait pas comment la personne injuriée a réagi » et dans Il s'est fait injurier, «on a l'idée qu'il s'est comporté de telle manière qu'on l'a injurié » (ibid.). La justification que ces auteurs donnent à cette allégation est la présence du «causatif» faire; en réalité, la valeur de cette construction ne se situe pas au niveau d'une supposée «responsabilité » du référent du sujet de la construction, mais se situerait au niveau aspectuel (cf. infra); le référent du sujet n'a pas plus de responsabilité dans Je me suis fait cambrioler que dans J'ai été cambriolé, surtout si l'on ajoute des indices de non-responsabilité : Je me suis fait cambrioler malgré le déclenchement de l'alarme que j'ai fait installer récemment. De même, la $N G F$ avance que : «se faire suppose une certaine responsabilité du sujet» (p. 107) en proposant un exemple ad hoc: «Monsieur Dupuis s'est fait licencier pour faute professionnelle», or, le SP pour faute professionnelle ici induit forcément une interprétation où le référent du sujet a effectivement une responsabilité, mais une fois le SP retiré, rien ne dit que le référent ait une quelconque responsabilité dans son licenciement.

C'est plutôt à un niveau aspectuel qu'il convient de présenter le passif en se faire aux apprenants, comme nous le verrons plus loin, car cet aspect n'est jamais mentionné explicitement et n'est donc pas ou très peu exploité dans les manuels de FLE. De plus, cette forme de passif n'est pas suffisamment mise à l'honneur, dans les manuels, alors que c'est une forme extrêmement vivante et fréquente de passif, surtout à l'oral. Ainsi, on trouve dans la $G E F-N D$, la formulation «J'ai été eue!» comme titre d'un texte dont les verbes sont à mettre au passif. Ce genre de phrase a très peu de chances d'être produit par un francophone, là où «Je me suis fait avoir!» est tellement banale! La tournure passive en se faire permet au verbe avoir de prendre le sens figuré de «tromper, abuser», alors que le passif «canonique» privilégie le sens propre du verbe (Le Bellec, 2014, p. 219), sans toutefois exclure la possibilité d'une lecture au sens figuré2 ${ }^{2}$

2. Voir la section 3.2 de cet article où cette caractéristique est développée. 


\section{3. Éléments de transposition didactique pour l'enseignement du passif}

Dans ce qui suit, on se focalisera sur les trois points qui présentent, selon nous, un enjeu majeur dans l'étude du passif en français et qui doivent être pris en compte dans la conception des outils pédagogiques en FLE : (1) la reconsidération de certains amalgames notionnels répandus du point de vue des usages des phrases passives, (2) la prise en compte des valeurs aspectuelles du procès et (3) les implications du choix de la préposition introduisant le complément d'agent.

\subsection{Phrase passive et phrase active, l'équation compromise!}

Bien que paraissant remplir, du point de vue référentiel, le même rôle, en tant qu' «agent» de l'action, complément d'agent et sujet, n'ont pas, du point de vue discursif, le même sens : si le sujet dans la phrase active est une donnée que l'on peut qualifier de relativement «neutre» (c'est l'«agent» unique de l'action en question), l'agent en par, dans la version passive, le présente, du point de vue de l'énonciateur, comme un agent «possible» parmi d'autres (Hamma, 2005, 2007a). Nous pourrions y voir ce que Leeman (1998, p. 20-22) appelle un effet de «contraste», opposant l'agent à un autre de manière «différentielle» : quand on énonce Max a préparé une salade, on ne fait que donner une information que notre interlocuteur demande ou que l'on juge pertinente compte tenu de la situation; dans ce cas, on part de ce qui est «connu» : le thème $\operatorname{Max}$ (un $N$ propre, donc référant à une personne connue par les interlocuteurs ou évidente selon le co(n)texte : il/elle/je, etc., le cuisinier/son mari, etc.) pour en dire quelque chose, le prédicat : a préparé une salade. Le prédicat est donc une donnée nouvelle que l'interlocuteur est censé ignorer ou que l'on présume telle, selon le principe d'«informativité».

Quant à la version passive de la phrase, on pose une situation différente, en quelque sorte, contraire à la première : c'est l'objet direct de la phrase active, ici une salade, qui est topicalisé dans la nouvelle phrase, et son thème (Max) est alors l'enjeu de l'acte de langage; c'est la donnée manquante (ignorée par l'interlocuteur et que l'on cherche à communiquer). Ce mécanisme peut expliquer, par exemple, pourquoi une phrase comme la suivante n'est pas naturelle ${ }^{3}$ :

3. Le fait qu'un énoncé peut être perçu comme non naturel recouvre, outre son sémantisme et sa grammaticalité, ce que nous avons désigné par «dicibilité» 
(7) a. ?Une salade a été préparée par Max.

La bizarrerie ici vient de l'emploi de l'article indéfini (une) qui implique que ce dont on parle n'est pas connu par l'interlocuteur. De fait, la simple substitution de l'article défini (la) ou du démonstratif (cette) - qui relève d'un «indexical» - à l'article indéfini (une) enlève la bizarrerie constatée :

(7) b. (La+Cette) salade a été préparée par Max.

Cette constatation permet d'expliquer aussi pourquoi l'exemple Paul a lâché la rampe (= «est mort»), emprunté à Leclère (1993, p. 10), n'admet pas la transformation passive, tout comme pour l'exemple suivant: *La rampe est lâchée par Paul. D'après l'auteur, il s'agit d'une «métaphore figée» (ibid.) ; mais on peut aller plus loin dans l'explication de ce blocage en lui donnant une justification en rapport avec les remarques précédentes : on a vu qu'il y a une sorte de mise en avant du thème de la phrase passive (on commence par évoquer un fait connu ou auquel le contexte de l'énonciation renvoie); or, partir de La rampe, le thème de la phrase passive, supposerait que l'on parle de quelque chose de «connu»/ «neutre», ce qui s'oppose à la nature «métaphorique» ou «figée» en question, d'autant plus qu'une interprétation littérale de cet exemple n'est pas exclue. Mais à défaut du rapport avec ce qui est «connu» par les interlocuteurs, cette structure donne lieu à un énoncé bizarre - fait corroboré par ce que Mejri (2002) appelle «rupture paradigmatique» dans certaines «séquences figées » du même type *une douche française (au lieu d'écossaise) *une fille manquée (au lieu de garçon).

Par ailleurs, le fait que le SN1 semble plus important du point de vue énonciatif, ici, est corroboré par le fait que la plupart du temps - et non toujours -, l'agent en par est supprimable; il s'agit d'une information de second degré : ce qui est mis en avant, c'est l'état résultant du procès (cf. l'utilisation de l'aspect accompli) ${ }^{4}$. Ce fait relevé ici est confirmé par certaines études, notamment celle de Wilmet (2000, p. 269) : dans Le carburateur a été démonté par Pierre (ibid.), c'est Le carburateur

(Hamma, 2007b); en d'autres termes, une phrase peut ne pas être naturelle alors qu'elle répond parfaitement aux deux premiers critères.

4. Néanmoins, si l'agent en par est exprimé, le passif peut alors avoir un sens processuel équivalent à l'actif : Le carburateur est démonté par Pierre équivaut à Pierre démonte le carburateur. 
qui est mis en valeur. Cette dimension subalterne de l'agent dans la phrase passive est sans doute l'un des arguments qui justifie les affinités que l'on peut établir entre les phrases passives sans complément d'agent et les phrases actives qui se construisent avec un sujet indéfini comme on (2) ou impersonnel comme il (3), conformément à ce qui a été présenté par le $N E d$ (cf. ci-dessus). Ainsi, passer de (1) à (2) ou à (3) semble ad hoc pour les raisons évoquées plus haut. En effet, le scénario mis en scène ne fait pas appel à une logique discursive de «contraste» : il n'est pas question d'opposer un agent possible à un autre; il s'agit tout simplement de donner une information concernant «la hausse des prix du carburant». L'utilisation du passif, en l'occurrence, met en avant l'aspect «accompli» du procès tout en topicalisant le prédicat à la forme active. Mais on verra, plus loin, que quand l'agent est exprimé et quand il est introduit par la préposition par, cela change la donne de manière sensible au niveau de la prise en charge de ce qui est annoncé par l'énonciateur.

Ces différentes remarques confortent ainsi nos hypothèses selon lesquelles la phrase passive ne véhicule pas tout à fait le même sens que la phrase active; le jugement contraire s'appuie, en fait, sur une vision «objectiviste» de la langue, en tant qu'elle décrit le monde et ne tient pas compte du fait que dans le système linguistique, le changement de forme n'est jamais gratuit, comme nous venons de le montrer. D'ailleurs, certaines phrases passives n'ont pas d'équivalent à la forme active et réciproquement, et d'autres impliquent une information complètement différente au niveau du procès : Ce tissu irrite la peau, exemple emprunté à Gross (2000, p. 25) n'est pas l'équivalent exact (abstraction faite de l'effet de topicalisation) de La peau est irritée par ce tissu. La première phrase - mais non la seconde - peut avoir une double lecture : une interprétation processive (cf. «Le tissu de la chemise que je porte m'irrite la peau» : état résultatif) et une interprétation à valeur de «vérité générale», décrivant l'une des «propriétés » de ce tissu et le résultat du procès irrite, quoique vrai, reste latent ( $«$ Je te déconseille ce tissu; il irrite la peau»), ce qui est à rapprocher de ce que Herslund (1999, p. 71) appelle «sens épistémique» ou «déontique» (avec le passif réfléchi) dans des exemples comme On va sourire car l'angoisse ne se partage pas, qui n'admet pas de lecture évènementielle.

La dualité «inconnu/connu» joue le même rôle dans les phrases passives en par. La préposition révèle que le locuteur, en (7a) et (7b), en apportant cette information nouvelle, vient préciser quelque chose qui ne semble pas aller de soi; imaginons par exemple que la situation 
donnée l'incite à intervenir, à rectifier une erreur observée sur le thème La salade (qu'on vient de manger + que tu trouves excellente) : son interlocuteur se met alors à le féliciter pour avoir préparé la salade en question et le locuteur, en toute honnêteté, précise que ce n'était pas lui qui l'avait préparée et qu'il fallait, ainsi, féliciter Max.

La question qui se pose alors est de savoir pourquoi c'est la préposition par (et parfois de) que la langue a choisie pour véhiculer ce type de rapport; mais avant d'aborder ce point, il nous semble nécessaire de traiter de la question de l'aspect dans les phrases passives/actives.

\subsection{L'importance d'intégrer le phénomène d'aspectualité}

Souvent la relation d'équivalence établie entre la version passive et sa contrepartie active banalise l'apport de l'aspect du procès et son incidence sémantico-pragmatique, comme l'illustrent les exemples suivants : Le vin est servi («résultat du procès») versus Le sommelier sert le vin («procès en cours de réalisation») (Carlier, 2002; Helland, 2002).

Cette différence aspectuelle entre la forme active et la forme passive est décelable exclusivement avec des verbes qui présentent, du point de vue de l'aspect lexical ou Aktionsart, un procès «télique», puisque l'interprétation d'un état résultant du participe passé n'est disponible que si l'action aboutit à un état «achevé» stable. Cependant, la présence du complément d'agent dans la phrase passive peut conférer au verbe une certaine processivité, en fonction de la préposition employée (cf. cidessous, de versus par), ce qui permet d'opposer le «passif processif» en (8a) au «passif adjectival» ou «passif d'état» en (8b) :

(8) a. La maison est construite par une firme suédoise (= passif processif) (Muller, 2000).

(8) b. La maison est construite en briques (= passif adjectival / état résultant) (ibid.).

En revanche, les verbes «atéliques», qui marquent donc un «état» ou une «activité», ne diffèrent pas, sur le plan aspectuel, de la version active, comme le montre le couple de phrases : Tout le monde apprécie Pierre versus Pierre est apprécié de tout le monde (Carlier, 2002, p. 42).

Notons, en outre, qu'avec les verbes «atéliques», la mise au passif est parfois difficile ou semble moins naturelle que dans la forme active, ce qui s'explique certainement par le fait que ces verbes servent exclusivement à l'aspect processif et ont donc de grandes difficultés à exprimer l'aspect résultant du procès, contrairement aux verbes «téliques », 
comme l'illustre l'exemple suivant : ?Toutes les jolies femmes qui passent sont regardées avec intérêt par Alain $(G E F)$.

Afin de révéler le caractère «processif» de la situation évoquée, on peut recourir au test avec être en train de qui permet de se rendre compte que le passif «canonique» est moins naturel avec certains verbes (notamment les verbes «atéliques»), contrairement au passif en se faire, où la locution s'insère plus naturellement, ce qui nous permettrait de penser que la construction en se faire pourrait pallier cette restriction aspectuelle pour ce type de verbes: Jean est en train de se faire agresser, plutôt que ?Jean est en train d'être agressé; Il est en train de se faire gronder par la maitresse, plutôt que ?Il est en train d'être grondé par la maitresse.

Outre la dimension pragmatico-discursive (cf. section précédente), il convient ainsi de prendre en considération la dimension aspectuelle dans l'étude de ce genre de construction, puisque la forme passive acquiert un aspect différent de celui de sa contrepartie active, du fait que le procès est présenté comme «accompli» (cf. la forme composée constituée de l'auxiliaire être suivi du participe passé). En revanche, c'est plutôt l'aspect «non accompli» qui caractérise la forme constituée de «se faire + infinitif». Or, cette opposition est très souvent évincée des descriptions des règles du passif et est rarement mise en avant dans les manuels de FLE. Pourtant, la forme se faire + infinitif est l'une des formes passives les plus récurrentes en français moderne.

Selon Spang-Hanssen (1967, p. 141), la construction en se faire remplace la forme passive canonique «quand cette dernière ne peut être employée pour marquer une action en cours $[\ldots]$; avec la construction en se faire, on exprime plus nettement l'aspect inchoatif ou l'idée d'un processus». En effet, lorsque le verbe aller (en tant que semi-auxiliaire du futur) est employé dans une périphrase verbale temporelle, le passif canonique est impossible, puisque la tournure se focalise normalement sur l'entrée dans le procès, ce que la phrase passive canonique ne peut pas, en principe, exprimer, étant donné qu'elle jette la lumière sur l'état résultant du procès. C'est donc la passive en se faire qui est utilisée dans de tels contextes, afin de suppléer à cette restriction aspectuelle :

(9) a. Tu vas te faire renverser par la voiture.

(9) b. ?Tu vas être renversé par la voiture.

Ce phénomène est corroboré par Novakova $(2008$, p.7) qui remarque une fréquence élevée de la construction en se faire après les périphrases aspectuelles renvoyant aux différentes «phases de réalisation» du procès, 
comme : commencer à, être en train de, finir de, etc., étant donné que ce type de construction peut renvoyer à des procès «imperfectifs» et être considérés à la fois au début, en cours ou à la fin de sa réalisation.

Notons, cependant, que la phrase passive en se faire ne concerne pas toutes les classes de verbes; cette construction privilégie certains verbes «ditransitifs» et certaines valeurs au «sens figuré» de certains verbes.

D'autre part, comme le souligne, entre autres, Spang-Hanssen, la construction en se faire permet de transformer l'objet indirect de la phrase active en sujet de la phrase passive, ce qui est impossible pour la passive canonique, comme dans :

(10)a. Les riches dames mûres se faisaient voler leurs bijoux par des gigolos argentins. (Spang-Hanssen, 1967, p. 144)

(10)b. *Les riches dames mûres ont été volées leurs bijoux.

(11)a. Éric Naulleau : il s'est fait cracher dessus hier chez Ruquier! (dontmiss.fr)

(11)b. *Il a été craché dessus hier chez Ruquier!

(12) a. Je me suis fait pirater mon compte. (silicon.fr)

(12) b. *J'ai été piraté mon compte.

La construction en se faire viendrait donc pallier l'impossibilité pour la passive canonique de topicaliser un argument qui reçoit normalement la fonction objet indirect ou le rôle de «destinataire». L'argument correspondant au «destinataire» dans ce type de construction ne peut être promu à la fonction «sujet» dans les phrases passives dites «canoniques »; c'est donc la forme en se faire qui se substitue à celles-ci.

L'opposition aspectuelle entre la passive en se faire et la passive canonique pourrait également expliquer le fait que certaines tournures tendent à la «lexicalisation», comme : se faire pincer, se faire avoir, se faire rouler, se faire prendre, etc., au lieu de être pincé, être eu, être roulé, être pris, etc., qui sont difficilement acceptables dans le sens «figuré» qu'elles prennent avec se faire. Pour ces verbes, le passif en être a tendance à évoquer un sens «propre», alors qu'avec la construction en se faire, on a plus souvent un sens «figuré». Ce phénomène provient notamment du fait que la construction en se faire est susceptible d'exprimer un procès en cours de réalisation par opposition aux passives canoniques, qui expriment, dans ce type d'exemples, plutôt l'état résultant du procès (Le Bellec, 2014). 
La passive en se faire permet donc de lever l'ambigüité entre deux acceptions d'un même verbe, plus spécifiquement avec des verbes pouvant être pris au sens «figuré» :

(13)a. ?Il a été pincé par la police.

(13) b. Il s'est fait pincer par la police.

Dans le premier exemple (13a), si l'on admet qu'il soit acceptable, on aurait un sens «propre», signifiant que la police a fait l'action de «pincer avec les doigts», alors que la seconde (13b) manifeste un sens «figuré», signifiant qu'il «s'est fait attraper» par la police.

À présent, nous allons nous intéresser aux constructions passives avec complément d'agent pour considérer de près les mécanismes sousjacents aux choix des prépositions (par ou de).

\subsection{Choix et interchangeabilité des prépositions : par versus de}

Dire que par, comme tête du «complément d'agent», peut toujours remplacer de qui est, lui, qualifié de «tour littéraire» ou d' «emploi archaïsant», masque deux réalités langagières importantes : chaque préposition est liée à un paradigme différent de procès (des verbes «statifs» pour de et des verbes «dynamiques» pour par) et dans les occurrences où les deux prépositions sont possibles, le sens de la phrase change sensiblement.

À ce propos, Spang-Hanssen (1963, p. 66-67) montre que les deux prépositions s'emploient presque avec tous les types de verbe, on a : Couvert (par la + de) neige; Noirci (par la + de) fumée; Connu (par + de) tous; Accablé (par la + de) douleur, honte; Détesté $($ par + de) tous; Aimé $(\mathrm{par}+\mathrm{de})$ tous. Toutefois, par nécessite un déterminant devant les N AGENTIFS (en ce que cette préposition apporte de façon différentielle une précision sur la personne qui fait l'action), contrairement à de. L'auteur note que, assez souvent, il suffit d'une légère insistance sur le verbe ou sur le complément d'agent confinant à des compléments de «manière », de «cause» ou de «matière » (dans le sens de «instrument»/«moyen») pour que de cède la place devant par. Les verbes pouvant se construire avec ce type de complément sont très variés comme les présente l'auteur : confinant à la «manière » : agiter, animer, assaillir, etc.; à la «cause» : agacer, amuser, choquer, écceurer, effrayer, etc.; à la «matière (moyen)» : baigner, border, cerner, couper, etc.; des verbes «d'accompagnement» : accompagner, précéder, suivre, escorter, etc.; des verbes «de sentiment» : adorer, aimer, apprécier, etc.; des verbes «de perception» : comprendre, connaitre, 
écouter, entendre, etc. Spang-Hanssen semble ainsi postuler que la différence entre par et de tiendrait à un «infléchissement sémantique» vers le moyen, la manière ou la cause avec par, et que l'on peut les employer indifféremment. Or, l'examen des propriétés formelles et distributionnelles de chacune de ces deux prépositions pourrait montrer qu'elles sont tout à fait divergentes et du point de vue syntaxique et du point de vue sémantique. Nous avançons, à cet égard, deux preuves incontestables :

$\mathbf{1}^{\text {re }}$ preuve : par suppose nécessairement un déterminant, ce qui n'est pas le cas pour de (Les maisons ont été couvertes de neige/*de la neige ${ }^{5}$ vs Les maisons ont été couvertes par la neige/*par neige). On peut se demander si l'on a affaire avec de à un véritable complément d'agent, puisque le retournement à l'actif n'est pas possible, le sujet devant forcément être introduit par un déterminant: *Neige a couvert les maisons vs De la neige a couvert les maisons. De plus, couverte de neige dans l'énoncé La maison est couverte de neige est plus proche dans son fonctionnement d'un modifieur adjectival que d'un procès dynamique; selon des critères distributionnels particuliers :

- On peut très bien l'introduire dans une énumération d'adjectifs (non déverbaux) :

(14)a. La maison est déserte, triste, couverte de neige, pleine d'ordures et par-dessus le marché elle est située à côté de l'autoroute.

(14)b. ?La maison est déserte, triste, couverte par la neige, pleine d'ordures et par-dessus le marché elle est située à côté de l'autoroute.

- On peut supprimer le verbe être dans les titres ou les dénominations :

(15) a. La maison (est $+\varnothing)$ couverte de neige.

(15)b. La maison (est + ?Ø) couverte par la neige.

Il en va de même pour les exemples (16-17) où alternent les prépositions de et par; l'emploi des deux prépositions donne lieu à des phrases grammaticalement bien formées et sémantiquement acceptables; toutefois, ces exemples, selon que l'on emploie de ou par, renvoient à des situations différentes :

5. On peut voir aussi dans l'absence de déterminant partitif (de la) après la préposition de une haplologie qui empêche deux de de se suivre (la préposition de et $d u$, de la, des). 
(16) Ce tableau est aimélestimé (de + par) tous.

(17) Léa est suivie (d'+ par) un psy.

Certains grammairiens parlent de «tour littéraire» quand de introduit un complément d' «agent». Ainsi, d'après Arrivé et coll. (1986, p. 178), beaucoup d'emplois modernes de cette préposition (de) doivent être interprétés comme des «archaïsmes». Riegel et coll. (1994, p. 436), quant à eux, justifient cela par un argument diachronique : «Alors qu'en français classique, la préposition de était largement majoritaire (pour introduire le complément d'agent), le français moderne tend à généraliser par qui est toujours substituable à de.» De même, la pensée de Gaatone (1998, p. 194) semble aller dans ce sens : «Les emplois de par recouvrent ceux de de, même si dans quelques cas de peut paraitre préférable, alors que de est exclu de la plupart des contextes où figure par.» (C'est nous qui soulignons.) Néanmoins, il ne s'agit vraisemblablement pas que d'une affaire de préférence ou de choix mais plutôt d'une contrainte linguistique qui s'impose selon l'acte ou l'effet langagier que l'on vise à produire. Si les deux prépositions de et par peuvent commuter - mais non «toujours» (cf. *Cette salade est préparée de Max; *Paul est tué de Léa; etc.) - il n'en va pas moins que le sens change selon que l'on utilise l'une ou l'autre. Ainsi, estimé dans Ce tableau est estimé de tous a le sens de «apprécié», «important», «de valeur», «qui compte pour tout le monde» et informe de l'une des propriétés du tableau; «estimé (de)» se comporte plus comme un adjectif («attribut du sujet» Ce tableau) et admet un adverbe d'intensité du type très : Ce tableau est très estimé de tous.

D'ailleurs les gloses qu'on en a données commutent toutes avec estimé de dans cet énoncé, alors que dans Ce tableau est estimé par tous, c'est le comportement des visiteurs dans un musée, par exemple, vis-à-vis de ce tableau, qui est mis en avant; le participe peut commuter ainsi avec «prisé», «évalué», «jugé» et rejoint par conséquent les verbes attributifs qui admettent un «attribut de l'objet» : J'estime ce tableau authentique/Ce tableau est estimé par tous (à sa juste valeur + cent mille euros + comme le meilleur tableau impressionniste).

Par ici donne au procès un aspect «dynamique», «actif» et rend l'insertion de l'intensifieur très bizarre (*Ce tableau est très estimé par tous à sa juste valeur) contrairement à de qui lui donne un aspect «statif», «résultatif». De même avec aimé de tous versus par tous : le sens avec de a trait à l'une des propriétés du sujet de la phrase comme dans Il est très populaire alors qu'avec par, le prédicat évoque la manière dont on témoigne de cet amour; avec Ce tableau est aimé par 
tous, ce sentiment est concrétisé par des gestes d'émerveillement, des expressions de fascination et des regards d'admiration, son succès auprès des photographes qui le mitraillent de flashs, etc. De fait, un tableau aimé de tous peut avoir une valeur, même quand le tableau n'est pas en face des admirateurs, il est aimé (c'est l'une de ses propriétés). Cependant, avec par SN2, on a un sens agentif, une action concomitante; aimer relève en fait d'une action concrète et non d'un simple «verbe de sentiment», ce qui explique la bizarrerie de ?Au Louvre, la Joconde est aimée par les peintres se trouvant à Pékin. La même analyse vaut aussi pour la paire Marie est suivie d'/par un psy : avec de, on localise Marie par rapport à un individu (un psy) : «il y a quelqu'un après elle dans une file, par exemple»; un psy peut commuter avec n'importe quel autre nom de métiers ou nom propre :

(18) Marie est suivie d' (un architecte + un peintre + un éboueur + Alfred).

et on peut même poursuivre, dans ce cas, puis de Fred (on a un emploi distributif); alors qu'avec par, on a plutôt le sens de «Marie suit un traitement chez un psychologue», ce qui rend la commutation avec d'autres noms de métiers, par exemple, difficile :

(19) ??Marie est suivie par (un architecte + un peintre + un éboueur + Alfred).

à moins d'attribuer à ces énoncés un autre sens : «Marie est poursuivie/ traquée », ce qui nous ramène au sens «processif» par opposition à la simple «localisation dans l'espace» (cf. aussi Alicia est entourée d'amis versus Alicia est entourée par des amis; dans le premier cas, on ne fait que prédiquer une propriété du sujet Alicia : «elle est sociable/ ouverte/épanouie socialement, etc.»; alors que dans le second exemple, le sens est plus «concret/physique/évènementiel»: «les amis d'Alicia forment un cercle et l'entourent réellement»).

$2^{\mathrm{e}}$ preuve : soit par exemple le couple (20) versus (21) :

(20) Paul arriva, accompagné de sa femme.

(21) Paul arriva, accompagné par sa femme. (Hamma, 2005)

La phrase (21) peut signifier que la femme de Paul a accompagné son mari en voiture. Autrement dit, elle l'a conduit à l'endroit où il arrive (et est repartie ensuite), ce qui n'est pas le cas de la phrase (20); on peut dire (22) mais non (23) :

(22) Paul arriva, accompagné en voiture par sa femme. 
(23) *Paul arriva, accompagné en voiture de sa femme.

Contrairement à de, qui parait se comporter davantage comme un modifieur de l'adjectif, la préposition par introduit un complément d'agent doté d'une certaine «autonomie» par rapport au procès et au sujet; en (21), sa femme est susceptible de désigner la femme en tant qu'elle agit (elle accompagne, c'est-à-dire conduit effectivement; elle n'apparait pas seulement à côté de Paul) : elle est venue le déposer, mais elle est repartie aussitôt.

De même pour Il est adoré de ses élèves, c'est-à-dire «il est aimé» (une propriété stative); cela s'oppose à elle est adorée par un homme à genoux, qui renvoie à un procès «actif»; un homme adore une femme comme on peut adorer les dieux, les statues. Cela suppose des rites particuliers, des actions effectives, concrètes («se mettre à genoux, produire certains gestes, etc.»); mais ce n'est pas parce qu'elle est adorée (dans le sens de «l'objet d'un culte») qu'elle est adorée (c'est-à-dire dans le sens de «l'objet d'une grande affection») : des peuplades peuvent «adorer» des monstres, c'est-à-dire leur rendre un culte, par peur et non par amour! Donc adorer avec par suppose une action concrète mais non forcément un sentiment d'«adoration/d'amour». Il en va de même pour l'exemple Ils sont bénis par le pape, mais non ??Ils sont bénis du pape alors que l'on dirait bénis des Dieux/de Dieu (plutôt que par Dieu). En effet, pour le pape, il s'agit, selon le savoir partagé, d'une action effective, d'un geste concret (on est libre d'accepter d'être ou non béni) contrairement au cas de Dieu dans le discours religieux, selon lequel c'est Dieu qui décide (on n'a pas le choix, on n'est pas autonome : on est béni ou non sans qu'on le veuille) et il ne s'agit pas d'une action effective, d'un geste concret comme pour le pape, mais d'une certitude intime liée à la foi que le sujet éprouve.

\section{Pistes pour le travail de transposition}

Dans le but de rendre compte des différents points évoqués supra et qui, rappelons-le, semblent faire défaut dans la plupart des méthodes de FLE consultées (cf. ci-dessus), il conviendrait de prévoir une étape de sensibilisation ou de découverte précédant celle d'entrainement. L'objectif de cette étape sera de faire prendre conscience aux apprenants des possibilités qu'offre la langue française pour l'expression du passif, à travers quelques exemples choisis, qui respectent non seulement le plan syntaxique et sémantique, mais aussi celui de la «dicibilité» (avec un 
sujet plutôt animé, défini, etc.; un procès plutôt dynamique et se prêtant à la passivation; un complément d'agent naturel qu'introduit la préposition adéquate en fonction de l'effet recherché). Le choix des phrases devrait tenir compte de la variété des emplois observables (il ne faudrait pas se limiter au passif périphrastique et étendre cela, entre autres, au passif en se faire).

L'étape suivante consistera à attirer l'attention des apprenants sur le fait que des analogies peuvent être établies entre les versions actives et passives oralement, sous forme d'un jeu de questions-réponses, mais sans brusquer les choses, ni tomber dans les automatismes trompeurs. Ainsi, on pourrait associer l'absence de l'agent à l'usage du pronom indéfini (cf. On) en évoquant les scénarios possibles (discrétion, ignorance, etc., de l'agent); on associera l'aspect «statif» à l'usage de la préposition $d e$, là où la préposition par intervient avec des procès dynamiques et volontaires.

Par ailleurs, l'utilisation des dessins ou des photographies pourrait permettre de souligner les différences d'aspect impliquées par le passage de la version active à la version passive et réciproquement : on présentera une photo représentant, par exemple, un sommelier qui est en train de servir le vin, pour faire comprendre qu'il s'agit d'un procès «en cours» pour la phrase active (Le sommelier sert le vin). En revanche, pour la version passive (Le vin est servi par le sommelier), on montrera plutôt un sommelier qui se tient juste à côté de la table sur laquelle se trouve un verre rempli de vin; il sera aisé, ainsi, de faire comprendre que, contrairement à l'exemple actif, au passif, on a plutôt un résultat, en particulier avec une suppression de l'agent et si l'agent est précisé, on pourrait évoquer la notion de «contraste». En effet, on pourrait s'attendre à ce que quelqu'un d'autre serve le vin et il se trouve que c'est le sommelier qui s'en charge; ce serait ainsi une information non banale qui mérite d'être communiquée, dans une situation de communication donnée.

\section{Conclusion}

Notre étude montre l'intérêt que présente la prise en compte des implications sémantiques et pragmatiques des phrases passives; en particulier, il est difficile d'établir une relation d'équivalence entre les formes actives et passives qui dépasse le seul effet de «mise en valeur» ou d'«emphase». Il existe en effet d'autres phénomènes qui entrent en jeu et qui ont autant d'intérêt, à savoir la notion de «contraste» établie 
entre les agents «possibles» et celle de la dualité «connu/inconnu». Nous avons pu voir également que la question de l'aspect est au cœur de l'étude de la relation passive-active et qu'il est, par conséquent, judicieux de tenir compte de ce type de considérations. Ainsi, nous avons vu que la forme concurrente en «se faire + Infinitif» que connait le passif «canonique» - et qui est, par ailleurs, absente dans la plupart des manuels de FLE, quoique très courante en français - permet d'expliquer de nombreux phénomènes discursifs que l'on ne peut soustraire aux cours de FLE sur le passif. Enfin, nous avons considéré le choix ou l'usage de la préposition appropriée (par ou de) dans la même optique discursive, afin de prouver que l'utilisation de l'une ou l'autre de ces deux prépositions n'est pas une simple question de «préférence » ou de «style»; les manuels scolaires qui s'inspirent essentiellement de la grammaire traditionnelle tendent, en effet, à neutraliser leur usage, ainsi que les incidences que chacune de ces deux prépositions peut avoir sur le sens d'une phrase au passif.

\section{RÉFÉRENCES BIBLIOGRAPHIQUES}

Arrivé, Michel et coll. (1986). La grammaire d'aujourd'hui : guide alphabétique de linguistique française. Paris : Flammarion.

CARLIER, Anne. (2002). Les propriétés aspectuelles du passif. Dans V. Lagae, A. Carlier \& C. Benninger (dir.), Temps et aspect : de la grammaire au lexique. Cahiers Chronos (vol. 10, p. 41-63). Amsterdam-New York : Rodopi.

GaAtone, David. (1998). Le passif en français. Paris-Bruxelles : Duculot.

Gross, Maurice. (2000). Sur quelques extensions possibles de l'appellation passif. Dans Le passif. Actes du colloque international Institut d'études romanes (Études romanes, vol. 45, p. 171-184). Université de Copenhague, Danemark.

Hamma, Badreddine. (2005). L'invariant sémantique de la préposition PAR à travers les distributions syntaxiques et lexicales (Thèse de doctorat, Université Paris 10-Nanterre).

Hamma, Badreddine. (2007a). La notion de «contraste» dans les locutions de type par N. Modèles linguistiques, 55(1), 77-92.

Hamma, Badreddine. (2007b). Philosophie et linguistique à la croisée des chemins? La problématique de l'indicible. Dans Actes du colloque Res Per Nomen (p. 125-132). Université de Reims.

Helland, Hans Petter. (2002). Le passif périphrastique en français moderne (Études romanes, vol. 50). Université de Copenhague, Danemark. 
Herslund, Michael. (1999). Les deux passifs du français. Dans Le passif. Actes du colloque international Institut d'études romanes (Études romanes, vol. 45, p. 71-82). Université de Copenhague, Danemark.

LE BELleC, Christel. (2014). La construction passive en se faire : une forme concurrente et complémentaire du passif canonique. Journal of French Language Studies, 24, 203-222.

LE Goffic, Pierre. (1970). Linguistique et enseignement des langues : à propos du passif en français. Apprentissage du français langue étrangère, Langue française, 8(1), 78-89.

Leduc-Adine, Jean-Pierre. (1977). Actif et passif des manuels. Sur les exercices de grammaire, Langue française, 33, 90-101.

Leeman, Danielle. (1998). Les circonstants en question(s). Paris : Kimé.

MeJRI, Salah. (2002). Le figement lexical : nouvelles tendances. Cahiers de lexicologie, 80, 213-225.

Novakova, Iva. (2008). La construction se faire + Vinf : analyse fonctionnelle. Dans Représentations du sens linguistique (IV). Helsinki, FI.

Rasmussen, Kirsten Wølch. (1996). La motivation du passif. Étude de corpus. SCOLIA, 8, 95-116.

Riegel, Martin, Pellat, Jean-Christophe \& Rioul, René. (1997). Grammaire méthodique du français. Paris : Presses universitaires de France.

SPANG-HANSSEN, Ebbe. (1963). Les prépositions incolores du français moderne. Copenhague : G.E.C. Gads.

SpANG-Hanssen, Ebbe. (1967). Quelques périphrases passives du français moderne. Dans Actes du $14^{\mathrm{e}}$ Congrès des romanistes scandinaves dédiés à Holger Sten (Revue Romane, numéro spécial 1, p. 139-147). Copenhague : Akademisk Forlag.

Wilmet, Marc. (2000). Du passif à la topicalisation ou pour changer de sujet. Dans Le passif. Actes du colloque international Institut d'études romanes (Études romanes, vol. 45, p. 265-276). Université de Copenhague, Danemark.

\section{Manuels de FLE consultés}

$\boldsymbol{E} \boldsymbol{G}=$ Caquineau-Gündüz, Marie-Pierre, Delatour, Yvonne, Jennepin, Dominique \& LeSAge-LAngot, Françoise. (2005). Les exercices de grammaire, B1. Hachette FLE.

$\boldsymbol{G E F}=$ Mimran, Reine, Poisson-Quinton, Sylvie \& Mahéo-Le Coadic, Michèle (2002). Grammaire expliquée du français. CLE International.

$\boldsymbol{G E F - N D}$ = Boulet, Roxane, Vergne-Sirieys, Anne, Poisson-Quinton, Sylvie \& Huet-Ogle, Célyne (2003). Grammaire expliquée du français. Exercices, niveau débutant. CLE International.

GPFNA = Boularès, Michèle \& FrÉrot, Jean-Louis. (1997). Grammaire progressive du français, niveau avancé. CLE International. 
$\boldsymbol{G P F}$-NI = GrégoIre, Maïa \& ThiÈvenAz, Odile. (2003). Grammaire progressive du français, niveau intermédiaire. CLE International.

$\boldsymbol{G P T}=$ ABry, Dominique \& Chalaron, Marie-Laure. (2006). La grammaire des premiers temps. Grenoble : PUG.

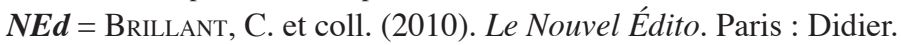

$\boldsymbol{N G \boldsymbol { F }}=$ Delatour, Yvonne, Jennepin, Dominique, Léon-Dufour, Maylis \&

TEyssier, Brigitte. (2004). Nouvelle grammaire du français. Cours de civilisation française de la Sorbonne. Hachette FLE. 